REDES- Revista hispana para el análisis de redes sociales.

Vol.5,\#4, Ene./Feb. 2004.

http://revista-redes.rediris.es

\title{
La dualidad y la agregación de categorías sociales ${ }^{1}$
}

\author{
Ronald L. Breiger ${ }^{2}$ - Department of Sociology. \\ University of Arizona (USA) \\ John W. Mohr - Department of Sociology. \\ University of California-Santa Barbara (USA)
}

\begin{abstract}
Resumen. La "dualidad" es una concepción del enlace micro-macro que implica que elementos correspondientes a dos niveles diferentes de la estructura (tales como personas y grupos, o -como en los datos que analizamos- identidades y prácticas) se co-constituyen mutuamente. Presentamos un algoritmo para la agregación de categorías sociales que son "duales" entre sí. Nuestro algoritmo es aplicable al estudio de datos en tablas de contingencia. Aplicamos nuestro algoritmo en un estudio de la construcción conjunta de identidades sociales (incluyendo etiquetas raciales y étnicas) y prácticas educativas en un contexto universitario.
\end{abstract}

Palabras clave: dualidad, agregación, co-constitución de identidades y prácticas.

\begin{abstract}
Duality" is a conception of micro-macro linkage that implies that elements at each of two different levels of structure (such as persons and groups, or-as in the data we analyze-identities and practices) co-constitute one another. We present an algorithm for the aggregation of social categories that are "dual" to each other. Our algorithm is applicable to the study of data in contingency tables. We apply our algorithm in a study of the joint construction of social identities (including racial and ethnic labels) and educational practices in a university context.
\end{abstract}

Keywords: duality, aggregation, co-constitution of identities and practices.

\footnotetext{
${ }^{1}$ Comunicación presentada en la Tercera Conferencia Europea de Análisis de Redes Sociales, LILNET: "Las relaciones micro-macro", Lille (Francia), 30-31 de mayo de 2002, traducida por Isidro Maya Jariego.

${ }^{2}$ Agradecemos a Ainhoa de Federico de la Rúa la invitación para publicar el artículo y su estímulo para terminarlo. Agradecemos a Michael Bourgeois, Linton Freeman, Alexis Ferrand, Emmanuel Lazega, Vincent Duquenne y Craig Rawlings por su consejo, y a los colegas de la Tercera Conferencia Temática Europea para el Análisis de Redes (celebrada Lille, Francia, en Mayo de 2002) que escucharon una versión previa de este artículo, presentada por el primer autor. La correspondencia relativa a este artículo puede remitirse a Ronald Breiger: Breiger@Arizona.Edu
} 
REDES- Revista hispana para el análisis de redes sociales.

Vol.5,\#4, Ene./Feb. 2004.

http://revista-redes.rediris.es

\section{La dualidad y la agregación de categorías sociales}

La "dualidad", una manifestación distintiva del vínculo micro-macro, se refiere a la co-constitución de elementos existentes en diferentes niveles de la estructura social (Breiger, 2000a). Por ejemplo, las personas de la elite política y económica están conectadas entre sí por medio de la incumbencia conjunta en posiciones de poder, incluso cuando las propias posiciones están conectadas por personas que mantienen pertenencias múltiples (Baena del Alcázar \& Pizarro, 1985). Teóricos sociales tan diversos como Coleman y Bourdieu han utilizado nociones de dualidad en sus formulaciones teóricas innovadoras sobre los acoplamientos micro-macro (véase la revisión en Breiger, 2000b, pp. 94-98 y 106-109). El trabajo de Mohr y colaboradores (Mohr, 1998, en prensa; Mohr y Duquenne, 1997; Mohr, Bourgeois y Duquenne, 2002; véase también Breiger, 2002b, Harcourt, 2002) pone énfasis en la dualidad de las prácticas locales y los símbolos culturales, las identidades y los roles globales, así como en la aplicación de métodos formales para el análisis de dichas prácticas.

En este artículo presentamos un algoritmo que sirve para implementar la agregación dual de las categorías de las filas y las columnas de una tabla de clasificación cruzada. Utilizamos el algoritmo para investigar la construcción conjunta de las categorías de identidad (incluyendo las etiquetas raciales y étnicas) y las prácticas educativas. La siguiente sección introduce el contexto de nuestros datos.

\section{Roles del discurso: categorías de identidad y prácticas organizacionales}

Mohr, Bourgeois, y Duquenne (2002) estudian varios cientos de organizaciones dentro del sistema de la Universidad de California (UC) en la denominada "era posterior a la acción afirmativa". (Desde 1995 el sistema de 
REDES- Revista hispana para el análisis de redes sociales.

Vol.5,\#4, Ene./Feb. 2004.

http://revista-redes.rediris.es

la UC ha prohibido la aplicación de los principios de la acción afirmativa en la admisión de estudiantes). Después de una breve revisión de su estudio, proporcionamos un nuevo análisis de los datos centrales del mismo, referente a la doble agregación de dos tipos diferentes de estructura (en este caso, las categorías de identidad y las prácticas de las organizaciones en relación con el principio de diversidad).

En respuesta a la prohibición legal de 1995, contra el uso de la acción afirmativa en la admisión de estudiantes, la Oficina del Presidente de la UC (OPUC) se orientó al desarrollo de mecanismos alternativos para preservar la diversidad étnica, racial y de género del alumnado. Desde 1998 se han gastado casi 350 millones de dólares de dinero público para financiar programas externos de la UC, entendidos como programas que utilizan personal y recursos universitarios para relacionarse con $y$, es de esperar, influir a los estudiantes de las escuelas públicas de primaria y secundaria para aumentar la probabilidad de que un alumnado diverso compita con éxito en la admisión a los campus de la UC. Los informes de 751 agencias y programas relacionados con estas actividades externas, de un rango que refleja todo el sistema de la UC, constituyen la fuente de datos del estudio de Mohr et al. (2002).

Como señalan los autores, el dilema fundamental en estos programas externos es que la diversidad racial, étnica y de género sigue asumiéndose plenamente como meta de la admisión de estudiantes, pese a que la acción afirmativa ha sido abandonada. Éstos escriben:

"Como cualquiera que haya sido miembro recientemente de un comité de admisión o de adjudicación de becas de la UC puede atestiguar, las distinciones aquí subrayadas prefiguran una situación discursiva nueva y más complicada para el personal de la universidad en la era post-acciónafirmativa... Nuestra meta es tomar alguna medición del sistema discursivo que opera en el campo organizacional de aquellas agencias de la UC encargadas de desarrollar actividades externas con estudiantes" (Mohr et al., 2002). 
REDES- Revista hispana para el análisis de redes sociales.

Vol.5,\#4, Ene./Feb. 2004.

http://revista-redes. rediris.es

¿Puede decirse que las categorías de identidad y las prácticas sobre diversidad que declaran las organizaciones se co-constituyen o se "construyen" mutuamente? Estos roles del discurso son el núcleo central del interés de Mohr et al. Para responder a esta cuestión los autores se centraron en las 279 organizaciones de la UC que, en sus declaraciones oficiales publicadas en un directorio de la OPUC, se referían a una categoría de identidad para el grupo diana y al mismo tiempo declaraban la utilización de prácticas específicas. Por ejemplo, una organización puede declarar programas dirigidos a estudiantes cuyo "primer idioma es el español" y proporcionar actividades de "enriquecimiento cultural". Puesto que la misma organización puede tener múltiples grupos clientes e implicarse en múltiples actividades, se obtuvo que las 279 organizaciones produjeron 620 enlaces de categorías de identidad con prácticas organizacionales, y estos enlaces están recogidos en la Tabla 1 (tomada de Mohr et al., 2002). Por ejemplo (véase la primera entrada de la Tabla 1, y la intersección de la fila 1 y la columna 1), "4" de las organizaciones proporcionan "mentores" a estudiantes pre-universitarios identificados como "asiáticos". 
REDES- Revista hispana para el análisis de redes sociales.

Vol.5,\#4, Ene./Feb. 2004.

http://revista-redes.rediris.es

Tabla 1. Tabla completa ( $12 \times 17)$ : I dentidades (filas) y Prácticas (columnas)

\begin{tabular}{|c|c|c|c|c|c|c|c|c|c|c|c|c|c|c|c|c|c|c|}
\hline & $\mathrm{mn}$ & Tu & $\mathrm{mo}$ & ap & Su & $\mathrm{Cl}$ & to & Ac & $\mathrm{pr}$ & ha & SS & ts & $\mathrm{cn}$ & fa & $\mathrm{CC}$ & sn & $\mathrm{sp}$ & $\begin{array}{l}\text { Total } \\
\text { counts }\end{array}$ \\
\hline ASIÁTICOS & 4 & 5 & 1 & 0 & 0 & 0 & 4 & 0 & 1 & 2 & 5 & 0 & 0 & 0 & 0 & 4 & 0 & 26 \\
\hline NEGROS & 1 & 0 & 0 & 0 & 1 & 0 & 2 & 0 & 2 & 1 & 1 & 0 & 0 & 0 & 0 & 2 & 0 & 10 \\
\hline CULTURA & 2 & 1 & 1 & 0 & 4 & 1 & 2 & 1 & 5 & 4 & 6 & 0 & 2 & 0 & 0 & 15 & 0 & 44 \\
\hline DIVERSO & 2 & 0 & 2 & 1 & 7 & 1 & 2 & 0 & 9 & 8 & 8 & 0 & 3 & 0 & 1 & 18 & 2 & 64 \\
\hline ESL & 2 & 4 & 2 & 0 & 7 & 0 & 5 & 3 & 14 & 18 & 23 & 1 & 5 & 2 & 0 & 18 & 2 & 106 \\
\hline ETNICO & 2 & 2 & 0 & 0 & 2 & 1 & 0 & 1 & 2 & 2 & 2 & 0 & 0 & 0 & 0 & 0 & 0 & 14 \\
\hline INMIGRANTE & 2 & 6 & 2 & 0 & 0 & 0 & 2 & 0 & 1 & 2 & 3 & 0 & 0 & 0 & 0 & 7 & 0 & 25 \\
\hline LATINO & 5 & 1 & 1 & 0 & 2 & 0 & 3 & 2 & 2 & 3 & 5 & 1 & 0 & 1 & 0 & 1 & 0 & 27 \\
\hline BAJOINGRESO & 5 & 12 & 5 & 1 & 10 & 7 & 4 & 7 & 5 & 11 & 7 & 1 & 1 & 1 & 4 & 2 & 1 & 84 \\
\hline MINORÍA & 10 & 7 & 3 & 3 & 8 & 3 & 10 & 9 & 10 & 8 & 17 & 0 & 7 & 1 & 0 & 4 & 1 & 101 \\
\hline INFRAREP & 9 & 6 & 5 & 1 & 11 & 4 & 5 & 8 & 8 & 12 & 8 & 2 & 4 & 0 & 2 & 1 & 0 & 86 \\
\hline URBANO & 4 & 3 & 2 & 1 & 2 & 0 & 0 & 1 & 1 & 8 & 6 & 1 & 0 & 0 & 0 & 2 & 2 & 33 \\
\hline Totales & 48 & 47 & 24 & 7 & 54 & 17 & 39 & 32 & 60 & 79 & 91 & 6 & 22 & 5 & 7 & 74 & 8 & 620 \\
\hline
\end{tabular}

Abreviaturas para los encabezados de las columnas (discursos sobre las prácticas): mentores (mn), tutores (tu), actividades motivacionales (mo), programa de aprendices (ap), programas de verano (su), cursos universitarios (cl), tours (to), orientación académica (ac), presentaciones (pr), actividades prácticas (ha), desarrollo de habilidades y estrategias (ss), preparación para la evaluación (ts), concursos y premios (cn), trabajo familiar (fa), capital cultural (cc), sensibilidad cultural (sn), deportes (sp). Fuente: Mohr, Bourgeois, and Duquenne (2002). 
REDES- Revista hispana para el análisis de redes sociales.

Vol.5,\#4, Ene./Feb. 2004.

http://revista-redes.rediris.es

La traducción de las descripciones del lenguaje natural sobre las identidades (tales como "la primera lengua es el español", "históricamente infra-representado", "urbano", "banda", "étnica y lingüísticamente diverso", "indochino", "asiático-americanos y americanos del Pacífico", "inmigrantes recientes") en las 12 categorías utilizadas por Mohr et al. (y reflejadas en nuestra Tabla 1) es un paso fundamental del análisis de los autores originales, que se llevó a cabo con un programa de análisis de textos escrito ex-profeso (Mohr et al., 2002). Estas 12 categorías de identidad se darán por supuesto en el presente análisis. Lo mismo es válido sobre las 17 categorías de prácticas organizacionales de las que se informa en la Tabla 1. Una descripción más detallada de cada una de estas categorías aparece en Mohr et al. (2002, Tabla 2).

\section{Agregación dual: un algoritmo}

En una red social, se dice que dos actores son estructuralmente equivalentes si tienen lazos idénticos con todos los demás actores de la red (véase, por ejemplo, las discusiones de Pizarro, 2000, y de Herrero, 2000). Consideremos que la Tabla 2 es una red dual, y consideremos a las categorías como actores (véase Douglas, 1986). Entonces podríamos afirmar, al respecto de dos filas (o al respecto de dos columnas), que éstas dos filas son estructuralmente equivalentes si cada fila tiene idénticos patrones de relación con todas las columnas (o con todas las filas respectivamente). Para datos en la forma de tablas de contingencia, dos filas tienen patrones idénticos de interacciones con todas las columnas si el modelo de independencia estadística se ajusta a estas dos filas (Goodman, 1981). Por tanto, afirmamos que el modelo de homogeneidad de Goodman (1981) proporciona un test de la equivalencia estructural para datos en tablas de contingencia. A continuación proporcionamos un ejemplo, y posteriormente utilizaremos dichos resultados en el algoritmo para la agregación dual que presentamos. 
REDES- Revista hispana para el análisis de redes sociales.

Vol.5,\#4, Ene./Feb. 2004.

http://revista-redes.rediris.es

Tabla 2. Cálculo ajustado de los modelos de independencia (con grados de libertad y valores chi-cuadrado)

\begin{tabular}{|c|c|c|c|c|c|c|c|c|c|c|c|c|c|c|c|c|c|}
\hline \multirow{4}{*}{$\begin{array}{l}\text { ÉTNICO } \\
\text { BAJO-INGRESO }\end{array}$} & \multicolumn{17}{|c|}{ A. Dos filas de la Tabla $1\left(\mathrm{gl}=16, \mathrm{G}^{2}=8.581\right)$} \\
\hline & $\mathrm{Mn}$ & Tu & mo & ap & su & $\mathrm{Cl}$ & to & $\mathrm{ac}$ & $\mathrm{pr}$ & ha & Ss & ts & $\mathrm{cn}$ & fa & CC & sn & $\mathrm{sp}$ \\
\hline & 1.0 & 2.0 & 0.7 & 0.1 & 1.7 & 1.1 & 0.6 & 1.1 & 1.0 & 1.9 & 1.3 & 0.1 & 0.1 & 0.1 & 0.6 & 0.3 & 0.1 \\
\hline & 6.0 & 12.0 & 4.3 & 0.9 & 10.3 & 6.9 & 3.4 & 6.9 & 6.0 & 11.1 & 7.7 & 0.9 & 0.9 & 0.9 & 3.4 & 1.7 & 0.9 \\
\hline & \multicolumn{17}{|c|}{ B. Tabla 1 , con ÉTNICO y BAJO-INGRESO combinados $\left(\mathrm{gl}=160, \mathrm{G}^{2}=274.806\right)$} \\
\hline & $\mathrm{Mn}$ & Tu & mo & ap & su & $\mathrm{Cl}$ & to & ac & $\mathrm{pr}$ & ha & Ss & ts & $\mathrm{cn}$ & fa & $\mathrm{CC}$ & sn & sp \\
\hline ASIÁTICOS & 2 & 2 & 1 & 0.3 & 2.3 & 0.7 & 1.6 & 1.3 & 2.5 & 3.3 & 3.8 & 0.3 & 0.9 & 0.2 & 0.3 & 3.1 & 0.3 \\
\hline NEGRO & 0.8 & 0.8 & 0.4 & 0.1 & 0.9 & 0.3 & 0.6 & 0.5 & 1 & 1.3 & 1.5 & 0.1 & 0.4 & 0.1 & 0.1 & 1.2 & 0.1 \\
\hline CULTURA & 3.4 & 3.3 & 1.7 & 0.5 & 3.8 & 1.2 & 2.8 & 2.3 & 4.3 & 5.6 & 6.5 & 0.4 & 1.6 & 0.4 & 0.5 & 5.3 & 0.6 \\
\hline DIVERSO & 5 & 4.9 & 2.5 & 0.7 & 5.6 & 1.8 & 4 & 3.3 & 6.2 & 8.2 & 9.4 & 0.6 & 2.3 & 0.5 & 0.7 & 7.6 & 0.8 \\
\hline ESL & 8.2 & 8 & 4.1 & 1.2 & 9.2 & 2.9 & 6.7 & 5.5 & 10 & 14 & 16 & 1 & 3.8 & 0.9 & 1.2 & 13 & 1.4 \\
\hline ÉTNICO + BAJOINGRES & 7.6 & 7.4 & 3.8 & 1.1 & 8.5 & 2.7 & 6.2 & 5.1 & 9.5 & 13 & 14 & 0.9 & 3.5 & 0.8 & 1.1 & 12 & 1.3 \\
\hline INMIGRANTE & 1.9 & 1.9 & 1 & 0.3 & 2.2 & 0.7 & 1.6 & 1.3 & 2.4 & 3.2 & 3.7 & 0.2 & 0.9 & 0.2 & 0.3 & 3 & 0.3 \\
\hline LATINO & 2.1 & 2 & 1 & 0.3 & 2.4 & 0.7 & 1.7 & 1.4 & 2.6 & 3.4 & 4 & 0.3 & 1 & 0.2 & 0.3 & 3.2 & 0.3 \\
\hline MINORÍA & 7.8 & 7.7 & 3.9 & 1.1 & 8.8 & 2.8 & 6.4 & 5.2 & 9.8 & 13 & 15 & 1 & 3.6 & 0.8 & 1.1 & 12 & 1.3 \\
\hline INFRAREP & 6.7 & 6.5 & 3.3 & 1 & 7.5 & 2.4 & 5.4 & 4.4 & 8.3 & 11 & 13 & 0.8 & 3.1 & 0.7 & 1 & 10 & 1.1 \\
\hline \multirow[t]{3}{*}{ URBANO } & 2.6 & 2.5 & 1.3 & 0.4 & 2.9 & 0.9 & 2.1 & 1.7 & 3.2 & 4.2 & 4.8 & 0.3 & 1.2 & 0.3 & 0.4 & 3.9 & 0.4 \\
\hline & \multicolumn{17}{|c|}{ C. Tabla 1 , tabla completa $\left(\mathrm{gl}=176, \mathrm{G}^{2}=283.387\right.$ ) } \\
\hline & $\mathrm{Mn}$ & Tu & mo & ap & su & $\mathrm{cl}$ & to & ac & $\mathrm{pr}$ & ha & Ss & ts & $\mathrm{cn}$ & fa & $\mathrm{CC}$ & sn & Sp \\
\hline ASI & 2 & 2 & 1 & 0.3 & 2.3 & 0.7 & 1.6 & 1.3 & 2.5 & 3.3 & 3.8 & 0.3 & 0.9 & 0.2 & 0.3 & 3.1 & 0.3 \\
\hline NEGRO & 0.8 & 0.8 & 0.4 & 0.1 & 0.9 & 0.3 & 0.6 & 0.5 & 1 & 1.3 & 1.5 & 0.1 & 0.4 & 0.1 & 0.1 & 1.2 & 0.1 \\
\hline CULTURA & 3.4 & 3.3 & 1.7 & 0.5 & 3.8 & 1.2 & 2.8 & 2.3 & 4.3 & 5.6 & 6.5 & 0.4 & 1.6 & 0.4 & 0.5 & 5.3 & 0.6 \\
\hline DIVERSO & 5 & 4.9 & 2.5 & 0.7 & 5.6 & 1.8 & 4 & 3.3 & 6.2 & 8.2 & 9.4 & 0.6 & 2.3 & 0.5 & 0.7 & 7.6 & 0.8 \\
\hline ESL & 8.2 & 8 & 4.1 & 1.2 & 9.2 & 2.9 & 6.7 & 5.5 & 10 & 14 & 16 & 1 & 3.8 & 0.9 & 1.2 & 13 & 1.4 \\
\hline ÉTNICO & 1.1 & 1.1 & 0.5 & 0.2 & 1.2 & 0.4 & 0.9 & 0.7 & 1.4 & 1.8 & 2.1 & 0.1 & 0.5 & 0.1 & 0.2 & 1.7 & 0.2 \\
\hline INMIGRANTE & 1.9 & 1.9 & 1 & 0.3 & 2.2 & 0.7 & 1.6 & 1.3 & 2.4 & 3.2 & 3.7 & 0.2 & 0.9 & 0.2 & 0.3 & 3 & 0.3 \\
\hline LATINO & 2.1 & 2 & 1 & 0.3 & 2.4 & 0.7 & 1.7 & 1.4 & 2.6 & 3.4 & 4 & 0.3 & 1 & 0.2 & 0.3 & 3.2 & 0.3 \\
\hline BAJO INGRESO & 6.5 & 6.4 & 3.3 & 0.9 & 7.3 & 2.3 & 5.3 & 4.3 & 8.1 & 11 & 12 & 0.8 & 3 & 0.7 & 0.9 & 10 & 1.1 \\
\hline MINORÍA & 7.8 & 7.7 & 3.9 & 1.1 & 8.8 & 2.8 & 6.4 & 5.2 & 9.8 & 13 & 15 & 1 & 3.6 & 0.8 & 1.1 & 12 & 1.3 \\
\hline INFRAREPRESENTADO & 6.7 & 6.5 & 3.3 & 1 & 7.5 & 2.4 & 5.4 & 4.4 & 8.3 & 11 & 13 & 0.8 & 3.1 & 0.7 & 1 & 10 & 1.1 \\
\hline URBANO & 2.6 & 2.5 & 1.3 & 0.4 & 2.9 & 0.9 & 2.1 & 1.7 & 3.2 & 4.2 & 4.8 & 0.3 & 1.2 & 0.3 & 0.4 & 3.9 & 0.4 \\
\hline
\end{tabular}


REDES- Revista hispana para el análisis de redes sociales.

Vol.5,\#4, Ene./Feb. 2004.

http://revista-redes.rediris.es

Fijándonos en la Tabla 1, consideremos la hipótesis de que las categorías de las filas ÉTNICO y BAJOINGRESO son estructuralmente equivalentes. La subtabla formada por estas dos filas es de un tamaño $2 \times 17$ (dos filas por 17 columnas), y el modelo habitual de independencia estadística, aplicado a esta subtabla, arroja 16 grados de libertad y un valor chi-cuadrado (ratio de probabilidad) de 8.581, como se muestra en el panel A de nuestra Tabla 2. Puesto que la chi-cuadrado es baja en relación con los grados de libertad, estimamos que estas dos categorías son estructuralmente equivalentes. Sumando estas dos filas de la Tabla 1 (una tabla que es $12 \times$ 17), obtenemos una forma reducida de tabla de tamaño $11 \times 17$. El modelo de independencia ajustada a esta forma reducida de tabla arroja $160 \mathrm{gl}$, y una chi-cuadrado de 274.806, como se muestra en el panel B de la Tabla 2. La independencia en la Tabla 1 por sí misma arroja $176 \mathrm{gl}$, y una chi-cuadrado de 283.387; véase el panel C de la Tabla 2. La relación entre estos resultados (Goodman, 1981) es muy importante para nosotros:

\begin{tabular}{|c|c|c|c|}
\hline Modelo & $\begin{array}{l}\text { Referencia en } \\
\text { la Tabla } 2\end{array}$ & Gl & $\begin{array}{l}\text { Chi-cuadrado } \\
\text { (Ratio de } \\
\text { probabilidad) }\end{array}$ \\
\hline Equivalencia estructural & panel $A$ & 16 & 8.581 \\
\hline $\begin{array}{l}\text { Independencia sobre una tabla } \\
\text { reducida } \\
(11 \times 17)\end{array}$ & panel B & +160 & +274.806 \\
\hline $\begin{array}{l}\text { Independencia de la tabla completa } \\
(12 \times 17)\end{array}$ & panel C & 176 & 283.387 \\
\hline
\end{tabular}

De cara a buscar una agregación de las categorías de las filas, nuestro algoritmo examina todos los pares de filas. Se encontró que ÉTNICO y BAJOINGRESO proporcionaron el mejor ajuste al modelo simple de independencia: el p-valor nominal sobre $16 \mathrm{gl}$ para una chi-cuadrado de 8.581 es .93 , y dicho valor se introduce sobre esas dos categorías de identidad de la Figura 1. (Nos referimos al p-valor como "nominal" porque buscamos empíricamente el mejor ajuste, y no estamos proporcionando un test formal de 
REDES- Revista hispana para el análisis de redes sociales.

Vol.5,\#4, Ene./Feb. 2004.

http://revista-redes.rediris.es

una hipótesis; por tanto, el p-valor es meramente una indicación descriptiva del grado de ajuste.) Entonces sumamos esas dos categorías de filas, y repetimos nuestro procedimiento con la tabla de $11 \times 17$ resultante. Las dos categorías con el mejor ajuste sobre esta tabla reducida son NEGROS y CULTURA $(p=.88)$, como puede verse en la Figura 1 . Continuamos con el procedimiento hasta que todas las categorías se han unido en una. Greenacre (1998) propuso un algoritmo relativamente similar para el análisis de correspondencias, que nosotros adoptamos aquí para los modelos loglineales y (específicamente) para los resultados de Goodman (1981).

Figura 1. Agregaciones duales: categorías de identidad

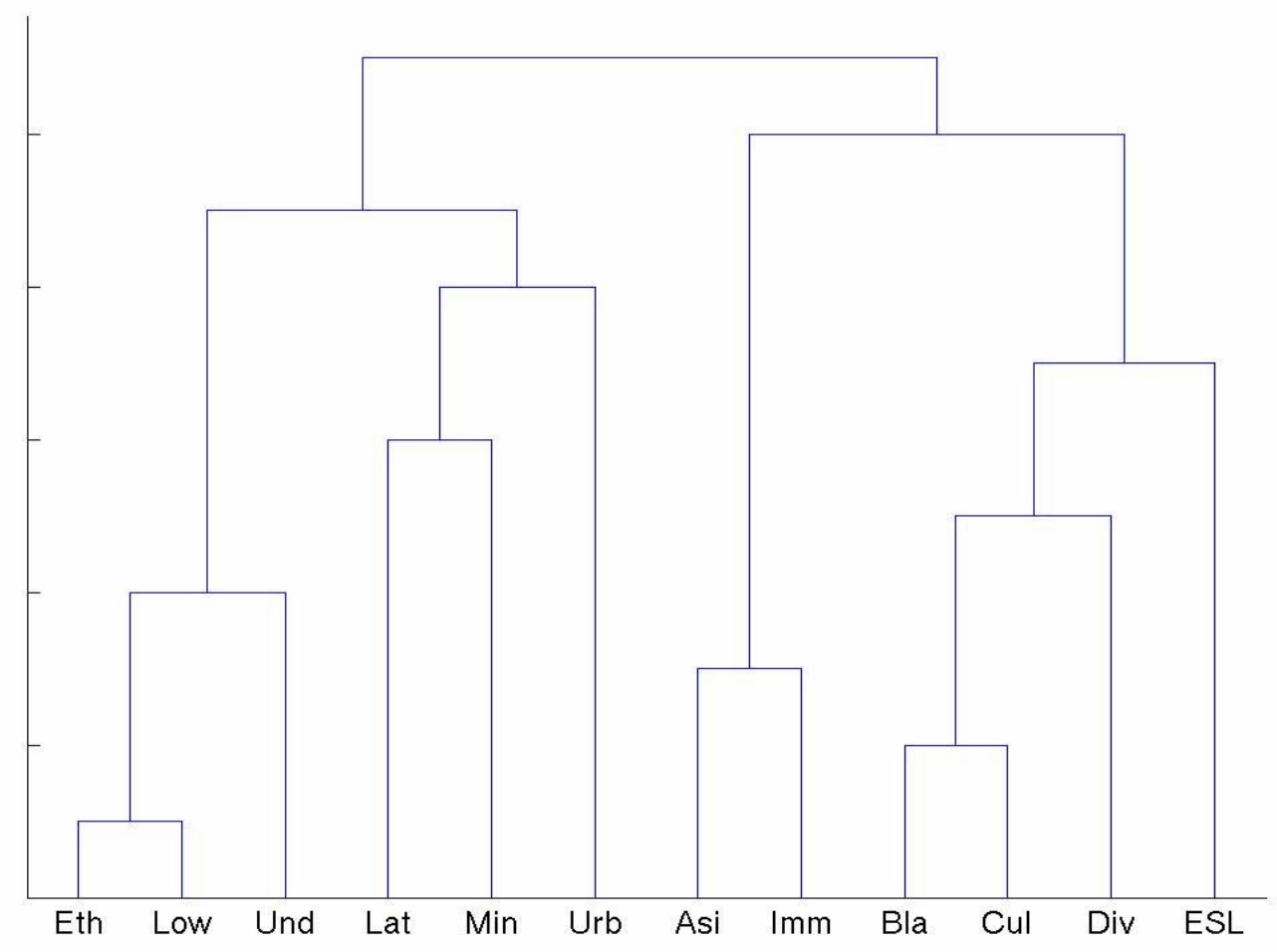


REDES- Revista hispana para el análisis de redes sociales.

Vol.5,\#4, Ene./Feb. 2004.

http://revista-redes.rediris.es

Figura 2. Agregaciones duales: prácticas. (Las abreviaturas se corresponden con las de la tabla 1)

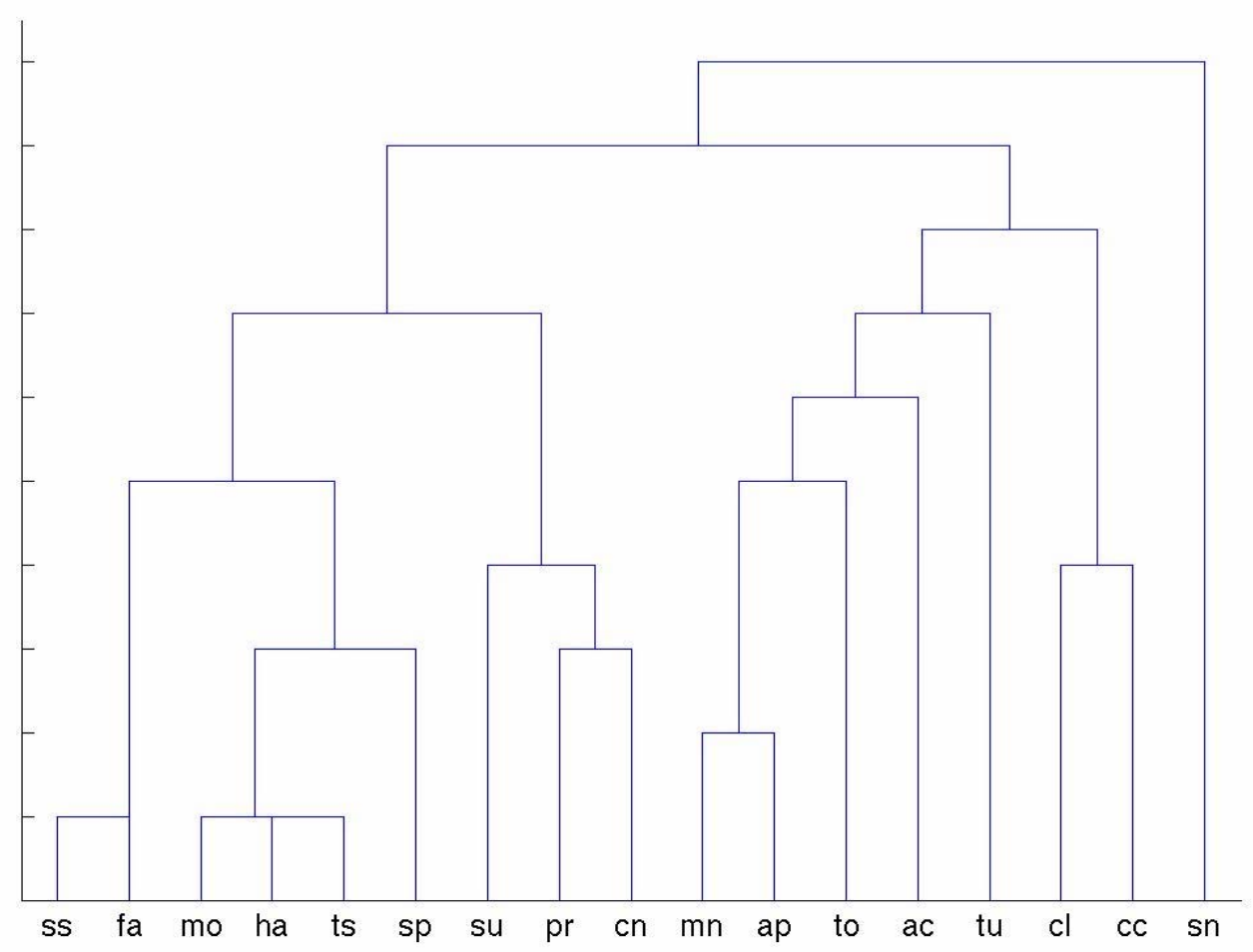

Nuestro algoritmo aplica el mismo procedimiento, separadamente, a las columnas de la Tabla 1. Adviértase en particular (en la Figura 2) cómo las prácticas pertenecientes a la sensibilidad cultural (con la etiqueta "sn") se resisten a ser agrupadas con cualquier otra categoría. Esta categoría (que incluye prácticas específicas tales como "diferentes esquemas de valores culturales", "tensiones raciales y culturales") es la que más difiere del resto de categorías sobre las prácticas.

Ponemos juntas las equivalencias de las categorías de identidad (Figura 1) y las prácticas (Figura 2) como se muestra en la Tabla 3. Ahí tenemos cinco categorías de las categorías de identidad estructuralmente equivalentes de la Figura 1, y seis categorías de las categorías de prácticas estructuralmente equivalentes de la Figura 2 . El tamaño de la tabla es por tanto $5 \times 6$. Esta es la 
REDES- Revista hispana para el análisis de redes sociales.

Vol.5,\#4, Ene./Feb. 2004.

http://revista-redes. rediris.es

"estructura de forma reducida" que se mantiene después de imponer la agregación dual que hemos descrito. El test de equivalencia estructural para estas cinco macro-identidades "cruzadas" con estos seis macro-conjuntos de prácticas arroja 156 grados de libertad y una chi-cuadrado de 130.97. Esto es, nuestro algoritmo ha identificado una equivalencia estructural substancial.

Tabla 3. Una agregación (tamaño $5 \times 6$ ) de la tabla de identidades y prácticas (Tabla 1)

\begin{tabular}{|c|c|c|c|c|c|c|c|c|c|}
\hline & Tu & $\begin{array}{c}\mathrm{ac}+ \\
\mathrm{ap}+ \\
\mathrm{mn}+ \\
\text { to }\end{array}$ & $\mathrm{Cl}+\mathrm{CC}$ & $\begin{array}{c}\mathrm{sp}+\mathrm{mo} \\
+\mathrm{ts}+ \\
\mathrm{ss}+\mathrm{fa}\end{array}$ & $\begin{array}{l}\mathrm{su}+\mathrm{pr} \\
+\mathrm{cn} \\
\end{array}$ & Sn & & $\mu_{1 \mathrm{i}}$ & $\mu_{2 i}$ \\
\hline $\begin{array}{l}\text { INFRAREPR + ÉTNICO } \\
+ \text { BAJOINGRESO }\end{array}$ & 20 & 43 & 18 & 57 & 43 & 3 & 184 & -.55 & .97 \\
\hline LATINO + MINORÍA & 8 & 42 & 3 & 41 & 29 & 5 & 128 & -.32 & .68 \\
\hline URBANO & 3 & 6 & 0 & 19 & 3 & 2 & 33 & .90 & .19 \\
\hline $\begin{array}{l}\text { NEGRO + CULTURAE + } \\
\text { DIVERSO + ESL }\end{array}$ & 5 & 23 & 3 & 81 & 59 & 53 & 224 & -.22 & $1 . \overline{30}$ \\
\hline $\begin{array}{l}\text { ASIÁTICOS } \\
\text { INMIGRANTE }\end{array}$ & 11 & 12 & 0 & 15 & 2 & 11 & 51 & 3.17 & .36 \\
\hline & 47 & 126 & 24 & 213 & 136 & 74 & 620 & & \\
\hline $\begin{array}{l}v_{1 j}= \\
v_{2 j}=\end{array}$ & $\begin{array}{l}.47 \\
1.68\end{array}$ & $\begin{array}{c}.24 \\
1.01\end{array}$ & $\begin{array}{l}-4.83 \\
-.19\end{array}$ & $\begin{array}{l}.23 \\
-.06\end{array}$ & $\begin{array}{l}-.21 \\
-.21\end{array}$ & $\begin{array}{l}.57 \\
-2.18\end{array}$ & & $\begin{array}{l}\varphi_{1}= \\
\varphi_{2}=\end{array}$ & $\begin{array}{l}1.36 \\
.46\end{array}$ \\
\hline
\end{tabular}

¿Cuál es la relación entre la tabla reducida (Tabla 3) y los datos originales (Tabla 1)? Puesto que estamos interesados en los campos organizacionales y culturales, tenemos un interés especial en trasladar estos campos a alguna forma de espacio multidimensional. (Breiger, 2000b elabora este argumento al respecto de los campos de Bourdieu, 2002; y Harcourt, 2002, basándose en gran medida en trabajos de Mohr y Breiger, hace un argumento similar respecto a los campos de prácticas sociales legalmente significativas.) Los modelos de asociación RC(M) de Goodman (por ejemplo, Goodman, 1996) proporcionan un contexto particularmente apto. El modelo general puede ser escrito log-multiplicativamente como: 
REDES- Revista hispana para el análisis de redes sociales.

Vol.5,\#4, Ene./Feb. 2004.

http://revista-redes. rediris.es

$\ln F_{i j}=u+u_{i}^{R}+u_{j}^{C}+\sum_{m=1}^{M} \phi_{m} \mu_{i m} v_{j m}$

donde la tabla de contingencia se va a representar en un espacio Mdimensional y donde los parámetros $\mu$ y $v$ son puntuaciones (para las filas y las columnas respectivamente) estimadas para cada dimensión. Los productos de estas puntuaciones (junto con una ponderación $\varphi$ para cada dimensión) representan la estructura de interacción multi-dimensional en la tabla, red de efectos de filas y columnas (véase, por ejemplo, Agresti, 2002). Sin el término multiplicativo en la ecuación (1), tenemos $\mathrm{RC}(0)$, idéntico al modelo de independencia simple para la tabla de contingencia.

Los modelos de 0, 1 y 2 dimensiones, todos ellos casos especiales de la ecuación 3, se ajustaron tanto a la tabla completa (Tabla 1) como a la tabla reducida (Tabla 3), y los estadísticos de ajuste están recogidos en la Tabla 4. El panel C de la Tabla 4 ilustra la partición de chi-cuadrado (Goodman, 1981) que es fundamental para nuestra aproximación de agregación dual. 
REDES- Revista hispana para el análisis de redes sociales.

Vol.5,\#4, Ene./Feb. 2004.

http://revista-redes. rediris.es

Tabla 4. Ajuste de modelos a la tabla completa y a la tabla reducida

A. El ajuste de los modelos seleccionados a la tabla completa (Tabla 1)

Modelo de asociación dimensional

A1. Asociación Nula (O)

Likelihood Ratio

A2. Asociación fila-columna, 1 dimensión (RC-1)

$176 \quad 283.39 \quad-848$

A3. Asociación fila-columna, 2 dims (RC-2)

150

161.97

$-802$

A4. Modelo RC-2 con ciertas categorías igualadas

126

88.85

$-722$

$162 \quad 140.42$

$-901$

B. El ajuste de los modelos seleccionados a la tabla reducida (Tabla 3)

\begin{tabular}{lll}
\hline Modelo dimensional de asociación & gl & $\begin{array}{l}\text { Likelihood Ratio } \\
\text { Chi-cuadrado }\end{array}$ \\
\hline B1. Asociación Nula (O) & 20 & 152.42 \\
B2. Asociación fila-columna, 1 dimensión (RC-1) & 12 & 55.7 \\
B3. Asociación fila-columna, 2 dims (RC-2) & 6 & 9.44 \\
\hline
\end{tabular}

C. Algunas relaciones entre estos modelos

$\mathrm{SE} \equiv$ Equivalencia Estructural $\equiv \mathrm{A} 1-\mathrm{B} 1$

$156 \quad 130.97$

$\mathrm{VR} \equiv$ Variación en la tabla reducida $\equiv \mathrm{B} 3$

VE $\equiv$ Variación en la tabla completa con puntuaciones igualadas $\equiv \mathrm{A} 4$

$=162=140.42$

El resultado final es que hemos llegado a un modelo (A4 en la Tabla 4) que tiene sólo 14 grados de libertad menos que el modelo de independencia, pero que se ajusta a los datos extraordinariamente bien. De modo substancial, este es un modelo bi-dimensional que tiene puntuaciones igualadas ( $\mu$ and $v$ ) en cada uno de los conjuntos estructuralmente equivalentes identificados en la Tabla 3. Estas son precisamente las puntuaciones $\mu$ y $v$ estimadas del modelo bi-dimensional ajustado a la tabla reducida (modelo B3 en la Tabla 4). La diferencia entre estos dos modelos (A4 y B3) es precisamente el test de la equivalencia estructural, como se ilustra en el panel $\mathrm{C}$. 
REDES- Revista hispana para el análisis de redes sociales.

Vol.5,\#4, Ene./Feb. 2004.

http://revista-redes.rediris.es

\section{Interpretación}

Pero, de modo substancial, ¿qué podemos aprender de todo esto? Las puntuaciones $\mu$ y $v$ estimadas de los modelos de interés se recogen en los márgenes de la Tabla 3, y estas puntuaciones se representan en la Figura 3.

Figura 3. Gráfico de los puntajes en dos dimensionesde las filas y columnas presentados en la tabla 3 (Puntajes de filas y columnas estimados para el modelo A4 y el modelo B3 de la tabla 4)

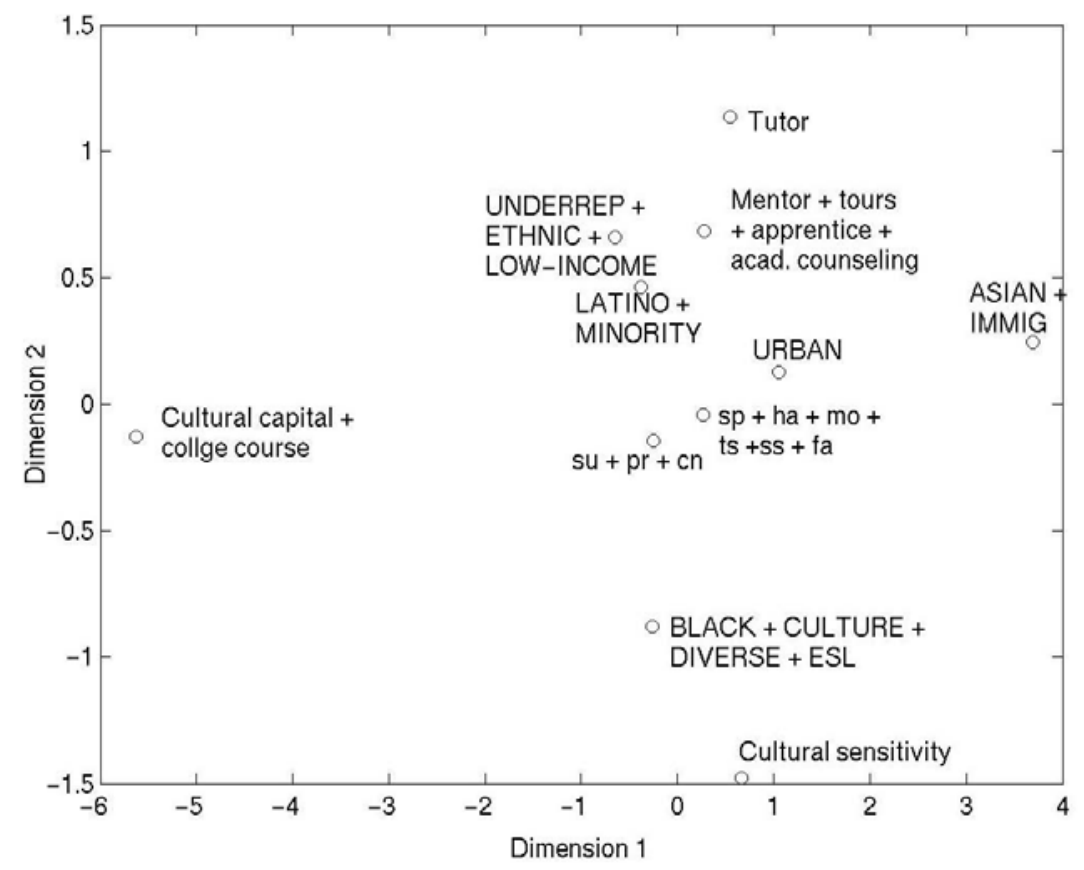

Al examinar la Figura 3, lo primero que llama la atención son las oposiciones que definen el espacio organizacional. Los intereses académicos (tutores, mentores, orientación académica, etcétera) dominan uno de los extremos de la dimensión vertical, mientras que la "sensibilidad cultural" define el extremo opuesto. Además, la sensibilidad cultural está altamente asociada con "Negro" y el resto de términos de la misma clase de equivalencia estructural, mientras que el extremo opuesto (académico) de la dimensión está muy asociado con asiáticos, inmigrantes, urbanos, latinos, minoría, infra-representado, étnico y grupos de bajos ingresos. Esto define lo que vemos como una "lógica de guía 
REDES- Revista hispana para el análisis de redes sociales.

Vol.5,\#4, Ene./Feb. 2004.

http://revista-redes.rediris.es

académica" en oposición a una "lógica de la diferencia", estando esta última centrada en la categoría de identidad "negro" y aquellas otras en la misma clase de equivalencia estructural (cultura, diverso, y ESL o "inglés como segunda lengua"). "Negro" y las identidades equivalentes son "opuestos" de guía académica, asociada en cualquier caso con las prácticas reconocedoras de la "diferencia".

La segunda oposición que llama la atención en la Figura 3 es la que existe entre asiáticos e inmigrantes (por un lado) y capital cultural y cursos universitarios (por el otro). Estas prácticas incluyen, por ejemplo, "visitas al museo", "sala de conciertos", "lecciones en el museo", "muestra de la vida universitaria" y "contexto universitario". Claramente, en este contexto institucional, se piensa (o se construye) que los asiáticos y los inmigrantes no "necesitan" capital cultural. Basándose en su interacción con tipos de identidades, el capital cultural es "diferente de" las prácticas de orientación académica y también de la sensibilidad cultural. En nuestra lectura, el nexo de capital cultural connota una "lógica de la desventaja" paternalista aplicada en particular a grupos latinos en el contexto contemporáneo de los Estados Unidos.

El artículo de Mohr et al. (2002), basándose en análisis Galois lattice, ha tenido éxito en la aplicación "de herramientas formales de las ciencias sociales para enfrentarse a la tarea interpretativa de comprender cómo los agentes organizacionales crean y consumen significado en... un espacio institucional". En este artículo hemos utilizado diferentes técnicas, centrándonos en la equivalencia estructural, el análisis dimensional, y la relación entre ambos. En cualquier caso, interpretamos la Figura 3 de un modo altamente compatible con el trabajo de los investigadores originales. Así, las relaciones formales entre las lattices y las representaciones dimensionales están recibiendo cada vez más atención por parte de los investigadores de redes (Pattison y Breiger, 2002), y sugiere la perspectiva de un conjunto de herramientas más unificado 
REDES- Revista hispana para el análisis de redes sociales.

Vol.5,\#4, Ene./Feb. 2004.

http://revista-redes.rediris.es

y auto-consciente para ayudar con los problemas de interpretación de las redes organizacionales.

\section{Conclusión}

"[L]as instituciones crean nuevas etiquetas, y las etiquetas crean nuevos tipos de personas. ... Para nosotros, la esperanza de la independencia intelectual es resistir, y el primer paso necesario en la resistencia es descubrir cómo el poder institucional se asienta en nuestra mente" (Douglas, 1986, pp. 92, 108).

Cuando una agencia de una universidad ofrece programas para facilitar que estudiantes diversos consigan la admisión en la universidad, dicha agencia está al mismo tiempo reflejando y construyendo un lazo entre las identidades de los estudiantes y los tipos de prácticas en los que se espera que se impliquen los actores sociales que son clasificados de determinado modo. En este artículo hemos presentado un algoritmo para ayudar a los analistas en la comprensión de cómo las instituciones llevan a cabo la clasificación (Douglas, 1986, pp. 91109). Esperamos que nuestra aproximación sea útil en el estudio de "la interdependencia de los actores y la acción a través de... niveles de agregación", que Lomi y Larsen (2001, pp. 6, 12) afirman que es "lo que convierte a las organizaciones en unidades significativas y relevantes de análisis".

Un argumento clave propuesto por "los teóricos de la práctica" tales como Giddens, Bourdieu, y de Certeau es que ni el mundo material (o el mundo de la acción) ni el cultural (el mundo de los símbolos) puede existir o estar coherentemente estructurado independientemente (Mohr y Duquenne, 1997, p. 309). Mohr y Duquenne han desarrollado un paralelismo entre el análisis de lattices y las preocupaciones de los teóricos de la práctica con las dualidades 
REDES- Revista hispana para el análisis de redes sociales.

Vol.5,\#4, Ene./Feb. 2004.

http://revista-redes.rediris.es

entre cultura y acción. En este artículo hemos presentado un algoritmo para la agregación dual que pretende ayudar en el análisis de los sistemas de enlaces entre las identidades y las prácticas. Parece que merece la pena crear puentes entre el análisis de redes y el trabajo reciente en el análisis de tablas de contingencia (por ejemplo, Goodman, 1996; Agreste, 2002). De especial interés (por ejemplo, en nuestra Figura 3 ) es la combinación del principio de dualidad de la red con modelos de tablas de contingencia que conllevan espacios multidimensionales dentro de los cuáles pueden identificarse campos organizacionales.

\section{Referencias bibliográficas}

Agresti, A. (2002), Categorical Data Analysis, 2nd ed., Wiley-Interscience, New York.

Baena del Alcazar, M., and N. Pizarro (1985), "The Structure of the Spanish Political Elite, 1939 - 1975," Research in Politics and Society, 1, 149-171.

Breiger, R.L. (2000a), "Control social y redes sociales: un modelo a partir de Georg Simmel," Politica y Sociedad, no. 33 (Enero-Mayo), 57-72.

Breiger, R.L. (2000b), "A Tool Kit for Practice Theory," Poetics, 27, 91-115.

Douglas, M. (1986), How Institutions Think, Syracuse University Press.

Goodman, L.A. (1981), "Criteria for Determining Whether Certain Categories in a Cross-Classification Table Should be Combined, with Special Reference to Occupational Categories in an Occupational Mobility Table," American Journal of Sociology, 87, 612-50.

Goodman, L.A. (1996), "A Single General Method for the Analysis of CrossClassified Data: Reconciliation and Synthesis of Some Methods of Pearson, Yule, and Fisher, and Also Some Methods of Correspondence Analysis and Association Analysis," Journal of the American Statistical Association , 91, 408-28. 
REDES- Revista hispana para el análisis de redes sociales.

Vol.5,\#4, Ene./Feb. 2004.

http://revista-redes.rediris.es

Greenacre, M.J. (1988), "Clustering the Rows and Columns of a Contingency Table," J ournal of Classification, 5, 39-51.

Harcourt, B.E. (2002), "Measured Interpretation: Introducing the Method of Correspondence Analysis to Legal Studies," University of Illinois Law Review, 2002, 979-1017.

Herrero, Reyes (2000), "La terminología del análisis de redes. Problemas de definición y de traducción," Politica y Sociedad, no. 33 (Enero-Mayo), 199216.

Lomi, A., and E.R. Larsen (2001), "Introduction," in A. Lomi and E.R. Larsen (Editors), Dynamics of Organizations: Computational Modeling and Organization Theories, MIT Press, Cambridge, MA, 3-34.

Mohr, J.W. (1998), "Measuring Meaning Structures," Annual Review of Sociology, 24, 345-70.

Mohr, J.W. (forthcoming), "The Discourses of Welfare and Welfare Reform," in M. Jacobs and N. Hanrahan (Editors), The Blackwell Companion to the Sociology of Culture, Blackwell, Oxford.

Mohr, J.W., M. Bourgeois, and V. Duquenne (2002), "How to Read an Institution: A Structuralist Approach," paper presented at the Sunbelt International Social Networks Conference, New Orleans (February).

Mohr, J.W., and V. Duquenne (1997), "The Duality of Culture and Practice: Poverty Relief in New York City, 1888-1917," Theory and Society, 26, 30556.

Pattison, P.E., and R.L. Breiger (2002), "Lattices and Dimensional Representations: Matrix Decompositions and Ordering Structures," Social Networks, 24, 423-44.

Pizarro, N. (2000), "Regularidad relacional, redes de lugares y reproducción social," Politica y Sociedad, No. 33 (Enero-Mayo), 167-198. 